\title{
Uma Proposta para Open Science em Ecossistemas de Software com Foco em Pesquisa e Educação em Engenharia de Software*
}

\author{
Francisco Victor da Silva Pinheiro ${ }^{1,2}$, Francisco Mateus dos Anjos Silva ${ }^{2}$, \\ Emanuel Ferreira Coutinho ${ }^{1,2}$
}

\author{
${ }^{1}$ Programa de Pós-Graduação em Computação (PCOMP) \\ ${ }^{2}$ Universidade Federal do Ceará (UFC) - Quixadá - CE - Brasil \\ \{victor.pinheiro.ce, mateus.16745\}@alu.ufc.br, emanuel.coutinho@ufc.br
}

\begin{abstract}
Resumo. Open Science pode ser definida como a combinação de vários movimentos e práticas com o objetivo de tornar o conhecimento científico abertamente disponível, acessível e reutilizável para todos. Diversas áreas da Engenharia de Software se encaixam nesse conceito de Open Science, sendo Ecossistemas de Software (ECOS) uma delas. ECOS pode ser definido como um conjunto de atores funcionando como uma unidade que interage com um mercado distribuído entre software e serviços. Uma carência da área de ECOS é a falta por modelos que possam ser utilizados principalmente para o ensino de ECOS. Uma vez que modelos de ECOS estejam disponíveis e padronizados, a difusão da área, extensão para novas pesquisas e colaboração é facilitada. $O$ objetivo desta pesquisa é iniciar estudos para a criação de uma base de modelos de ECOS aberta que permita a colaboração entre pesquisadores de ECOS tanto em pesquisas quanto para o ensino.
\end{abstract}

\section{Introdução}

Para a UNESCO, Open Science é definida como um construto inclusivo que combina vários movimentos e práticas com o objetivo de tornar o conhecimento científico abertamente disponível, acessível e reutilizável para todos, para aumentar as colaborações científicas e o compartilhamento de informações em benefício da ciência e da sociedade, e para abrir os processos de criação, avaliação e comunicação do conhecimento científico a atores sociais além da comunidade científica tradicional [UNESCO 2021]. Esta definição inclui todas as disciplinas científicas e aspectos das práticas acadêmicas, incluindo ciências básicas e aplicadas, naturais, sociais e humanas, e se baseia nos seguintes pilares principais: acesso aberto ao conhecimento científico, infraestruturas de ciência aberta, comunicação científica aberta, engajamento aberto de atores sociais e diálogo aberto com outros sistemas de conhecimento.

Nesse contexto, diversos tipos de sistemas e aplicações da Engenharia de Software (ES) se encaixam nesse conceito de Open Science. Diversas áreas podem se beneficiar, tanto em pesquisas em baixo nível, ou mais próximas a código, quanto pesquisas em um nível mais alto, como em Ecossistemas de Software, Sistemas de Sistemas e Sistemas Intensivos de Software.

\footnotetext{
*Os artefatos de revisão aberta por pares deste artigo estão disponíveis em https: / / zenodo.org/ communities/opensciense2021
} 
Ecossistemas de Software (ECOS) podem ser definidos como um conjunto de atores funcionando como uma unidade que interage com um mercado distribuído entre software e serviços [Jansen et al. 2009]. Estas relações são apoiadas por uma plataforma tecnológica ou por um mercado comum e realizadas pela troca de informações, artefatos e recursos. Manikas (2016) atualizou a definição de ECOS como sendo: "a interação do software e ator em relação a uma infraestrutura tecnológica comum, que resulta em um conjunto de contribuições e influencia direta ou indiretamente o ecossistema".

Uma carência relatada na literatura para a área de ECOS é a escassez de modelos que possam ser utilizados principalmente para o ensino de ECOS [Coutinho et al. 2017b][Coutinho et al. 2018]. Mesmo podendo ser modelado em diversas notações, como UML (Unified Modeling Language) e BMPN (Business Process Model and Notation), normalmente se modela um ECOS com a notação SSN (Software Supply Network) [Boucharas et al. 2009]. Uma vez que modelos de ECOS estejam padronizados e disponíveis, a difusão da área, extensão para novas pesquisas, colaboração e maior conhecimento sobre plataformas centrais ou de interesse do ECOS é facilitada.

Para a educação em ECOS, um repositório ou base de modelos padronizada e aberta seria de grande valia, pois possibilitaria para docentes exemplos, estudos de caso, pesquisas associadas e material para aulas. Padronizar a notação dos modelos também auxilia no ensino, pois os formatos são bem variados (camadas, redes sociotécnicas, grafos, diagramas de classes, etc). Para alunos, o aprendizado ficaria mais rico devido a vários modelos, diversos níveis de abstração de ECOS e exemplos de ECOS reais. Por fim, para a pesquisa, os modelos podem ser mantidos por uma ferramenta específica para modelagem de ECOS, e que exporte os modelos em formatos que possam ser lidos ou explorados por pesquisadores, devidamente armazenados em bases de dados abertas. O objetivo desta pesquisa é iniciar estudos para a criação de uma base de modelos de ECOS aberta que permita a colaboração entre pesquisadores de ECOS tanto em pesquisas quanto para o ensino. Seu foco é direcionado para pesquisadores em ECOS e quem deseja incorporar ECOS no ensino de ES.

\section{Ações para Motivar Open Science em ECOS}

Esta seção descreve algumas iniciativas para abordar Open Science em ECOS.

\subsection{Como Prover Open Science em ECOS?}

A criação de modelos de ECOS auxilia na concepção, evolução e maturação dos conceitos de ECOS. Ao pô-los em prática, por meio da criação do modelo, observando os relacionamentos simbióticos entre os atores do ECOS, as trocas de artefatos e recursos, uma visão geral e global sobre o desenvolvimento de sistemas, com o foco em aspectos sociais e de evolução se torna possível. Outro ponto importante em relação a modelos é a disponibilização, para que a comunidade tenha acesso e entenda o que está sendo produzido, além de possuir uma maior abrangência e visão global.

Ferramentas que auxiliem tanto na modelagem quanto na disponibilização de modelos são de relevante importância para o crescimento e disseminação da comunidade, possibilitando uma maior abrangência em pesquisas. Uma ferramenta de criação de modelos de ECOS possibilita a padronização, utilizando a notação de maneira correta, além de auxiliar no entendimento dos conceitos de ECOS por meio da notação sugerida. Uma 
vez que existam ferramentas que disponibilizem artefatos de ECOS (modelos ou dados) de maneira aberta, com o uso a quantidade de exemplos, modelos e estudos de caso se tornam mais conhecidos, disseminando a área e o domínio associado.

\subsection{Como Colaborar e Expandir Open Science em ECOS?}

Uma vez que existam meios de prover Open Science em ECOS, alguma estratégia para sua evolução deve ser planejada. Por evolução pode-se entender como o crescimento da plataforma de Open Science em ECOS, e como colaborar para que dados relacionados ao ECOS possam continuamente ser utilizados e ampliados. Assim, novas informações podem ser adicionadas, seja em conteúdo como novas funcionalidades ou atributos, seja por modelos. À medida em que novos elementos são adicionados, o ambiente de Open Science em ECOS amplia suas possibilidades de uso e de acesso pela comunidade.

Indiretamente, com a evolução de ferramentas de modelagem ou de disseminação de ECOS, pode-se colaborar com a expansão do Open Science na área, pois tais ambientes naturalmente armazenam dados sobre o ECOS. E a definição de atributos ou metadados associados aos modelos que serão utilizados nas ferramentas permite que os ambientes possam gerar bases de dados de modelos de ECOS para os mais diversos fins, como mineração de dados, medição da saúde do ECOS, etc. Por exemplo de metadados tem-se dados de cada elemento do modelo (nome, descrição, qualidade, etc). Como a utilização de notações para padronizar modelos (visual ou dados) ocorre a colaboração para que a comunidade possa ampliar seu uso. A incorporação da padronização dos modelos também colabora a disseminação, adição de conteúdo, e possibilidade de integração com outros ambientes. Essa colaboração ocorre principalmente pela capacidade de poder discutir sobre um modelo na mesma linguagem, e para comparar modelos.

A definição de papéis tanto nas ações de criação, colaboração/expansão e uso da base de modelos seria outra forma como colaborar. Por exemplo, quem insere os modelos na base, quem colabora com a expansão dessa base, quem são os usuários dos modelos. A ideia de administrador, autor, colaborador e demais papéis pode ampliar a pesquisa.

Por fim, a divulgação de ferramentas abertas de ECOS e repositórios de dados sobre modelos de ECOS possibilitaria além da disseminação da área, a colaboração em pesquisas. Ferramentas em um sentido mais amplo, experiências de ferramentas, sua utilização em sala de aula.

\section{Resultados Preliminares e Direcionamentos Futuros}

Um exemplo de como iniciar estudos de Open Science em ECOS seria pela disseminação de modelos de ECOS. Em geral, modelos de ECOS podem ser construídos em diversas notações, como UML e BPMN. Porém, especificidades de ECOS não são atendidas, ou são melhores direcionadas pela notação SSN [Boucharas et al. 2009]. Seguindo essa linha de raciocínio, onde modelos de ECOS na notação SSN podem ser construídos e distribuídos para a comunidade de ES, esta seria uma motivação inicial de Open Science em ECOS. Como ideias iniciais, seguir os elementos do modelo SSN e relacionamentos. Isso está implementado em uma ferramenta do grupo de pesquisa dos autores, porém não publicada. Atualmente a ferramenta está em refinamento, e já foi aplicada em sala de aula. Existem modelos na literatura abertos, vários publicados em artigos (por exemplo, o AVA SOLAR [Coutinho et al. 2017a] e o ECLIPSE [Mizushima and Ikawa 2011]). Entretanto 


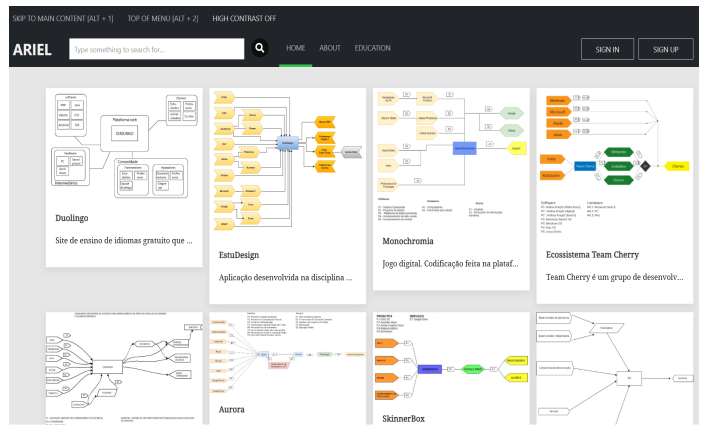

(a)

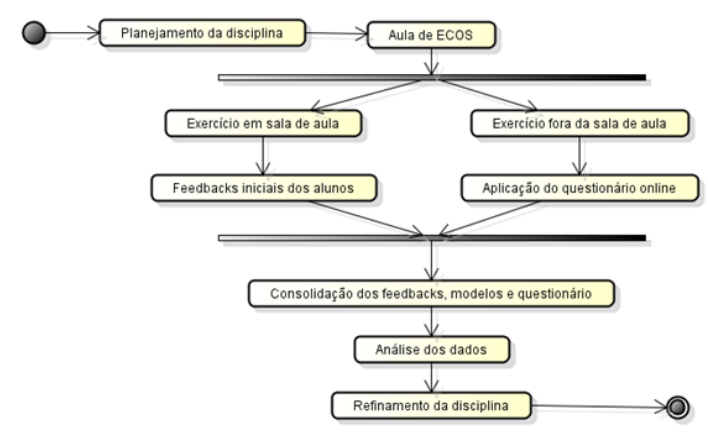

(b)

Figura 1. (a) Ferramenta desenvolvida [Alencar et al. 2020] e (b) Metodologia proposta para ensino de ECOS em ES [Coutinho et al. 2019]

eles estão espalhados, em diversos níveis e estilos, e não necessariamente com SSN. Uma forma de iniciar a base seria catalogar modelos já existentes na literatura, mesmo que não padronizados, e utilizá-la no ensino de ECOS. As limitações das ferramentas atuais estão na falta da capacidade de modelagem SSN.

Em termos práticos, algumas ferramentas específicas para cadastro e modelagem de ECOS foram propostas na literatura [Coutinho et al. 2019] [Alencar et al. 2020]. A Figura 1(a) ilustra uma das versões da ferramenta desenvolvida. A Figura 1(b) ilustra uma abordagem de utilização da ferramenta em sala de aula. Porém, ainda há necessidade de adequação para que melhor atenda aos pilares da Open Science: acesso aberto ao conhecimento científico, infraestruturas de ciência aberta, comunicação científica aberta, engajamento aberto de atores sociais e diálogo aberto com outros sistemas de conhecimento [UNESCO 2021]. Elas não modelam um ECOS, e sim utilizam imagens desenvolvidas em outras ferramentas de edição gráfica. Há retrabalho, não há possibilidade de alterar um modelo, o que não é produtivo. Quanto aos pilares, os ambientes e infraestrutura de ciência aberta não estão disponíveis, o que impede o engajamento.

$\mathrm{Na}$ literatura não foram identificadas ferramentas próprias para SSN. A maioria dos modelos são imagens elaboradas em editores gráficos. É necessário que esta busca sempre seja atualizada. Como adaptações, funcionalidades básicas para permitir inserir, editar, mover e redimensionar elementos, porém o que seria necessário é inicialmente a disponibilização dos componentes SSN, relacionamentos específicos e a semântica associada. Além disso, deve possibilitar o acesso e compartilhamento dos modelos para a comunidade. Uma ferramenta com essas características está em refinamento no grupo de pesquisa dos autores, porém ainda não publicada. Os modelos são visuais, então a extensão das ferramentas para a adição de atributos do modelo, tanto quantitativos quanto qualitativos, promoveria a criação de uma base de dados ou repositório de modelos. Por exemplo, dados de cada elemento do modelo (nome, descrição, qualidade, complexidade, etc). Qualitativo com opções de texto livre para colaboração, comentários e sugestões da comunidade. Esses dados em sendo exportados, facilitariam o uso e integração a outras ferramentas, implicando em novas ferramentas, colaborações e pesquisas.

Ao focar no domínio educação em ES, alguns trabalhos na literatura apontaram carências por modelos e exemplos de ECOS, tanto visuais quanto dados associados [Coutinho et al. 2018][Coutinho et al. 2019][Alencar et al. 2020]. Ao existir uma 
infraestrutura que possibilite o armazenamento e disseminação desses modelos, para a educação em ES isso implicaria em material para docentes planejarem aulas de conceitos e práticas em ECOS, modelagem de plataformas centrais em SSN e ampliação da base de dados de modelos. Consequentemente isso elevaria a qualidade e participação nas aulas, podendo extrapolar ECOS para as demais macro-atividades de ES.

Nesta pesquisa não se focou em ECOS na indústria, mas existem muitos modelos genéricos de ECOS para diversas plataformas e empresas de interesse, diretamente relacionadas à indústria. Por exemplo Google, Eclipse e Apple. O modelo pode ter o nível de abstração que for desejado. Muitos modelos informam apenas parte de um ECOS, uma integração, uma relação em estudo. É decisão de quem modela. Manikas e Hansen (2013) apresentaram uma lista diversos modelos de ECOS nos mais variados níveis.

A educação em ECOS se beneficiaria muito com recursos Open Science. Modelos acessíveis, dados de ECOS variados e ferramentas de ECOS podem colaborar para o ensino e relacionamento de ECOS com demais áreas da ES. Popular uma base de dados de modelos é um ideia. Iniciativas em sala de aula com alunos modelando ECOS em SSN ocorreram para esse fim, mas foram prejudicadas pela falta de ferramenta adequada. Para importação e exportação de modelos, alguns estudos já foram conduzidos no grupo de pesquisa, sendo passos importantes para integração. A base irá crescer com o uso, então modelos variados (diferentes níveis de abstração e complexidade) surgirão.

Outro ponto é que a criação de bases de modelos de ECOS para Open Science podem ser bastante similar em diversos contextos e domínios. O trabalho pode caminhar para a definição de um framework para bases Open Science e os contextos seriam características a serem observadas nesse framework ou em uma "nstância" dele.

Por fim, a saúde do ECOS é um aspecto que também pode se beneficiar com a Open Science. Santos et al. (2014) descreveram a saúde em ECOS como sua capacidade de permanecer estável e expandir, atendendo às demandas diárias. Iansiti e Levien (2004) apresentam que a saúde em ECOS é a capacidade de desenvolver o ecossistema e mantê-lo atraente para todos os membros da comunidade. Eles também fornecem três indicadores de saúde: robustez, produtividade e criação de nicho. Nesse contexto, Open Science em ECOS pode explorar a saúde tanto nesses três indicadores quanto nos demais aspectos relacionados a ECOS, como modelagem, visão global e impactos.

\section{Conclusão}

Este trabalho é uma iniciativa para estudos em Open Science em ECOS. Existem muitas possibilidades para pesquisa na área e diversas formas de colaboração, tanto para educação em ES, quanto para modelagem de sistemas. Há a necessidade de aprofundar o conceito de Open Science, seus princípios e como pô-lo em prática, e assim entender como ele pode ser incorporado a ECOS e consequentemente para auxiliar no ensino de ECOS. A ferramenta já desenvolvida pelo grupo de pesquisa, porém ainda não publicada já seria um resultado emergente. Porém ela ainda não atende a requisitos de OpenScience. Ela já foi aplicada em sala de aula algumas vezes. Como próximos passos, há a intenção de aprofundar mais conceitos de Open Science, para poder alinhar melhor com os conceitos de ECOS e possibilitar a união das duas áreas de maneira mais adequada, e adequar alguma ferramenta existente para que possa disponibilizar modelos de ECOS de maneira aberta e colaborativa, de forma a apoiar a pesquisa e educação em ECOS. 


\section{Referências}

Alencar, I. R., Coutinho, E. F., Moreira, L. O., and Bezerra, C. I. M. (2020). A tool for software ecosystem models: An analysis on their implications in education. In Proceedings of the XXXIV Brazilian Symposium on Software Engineering, SBES 2020.

Boucharas, V., Jansen, S., and Brinkkemper, S. (2009). Formalizing software ecosystem modeling. In Proceedings of the 1st International Workshop on Open Component Ecosystems, IWOCE '09, pages 41-50, New York, NY, USA. ACM.

Coutinho, E., Santos, I., Moreira, L., and Bezerra, C. (2018). Um estudo preliminar de ecossistemas de software na disciplina engenharia de software. Anais do Workshop de Informática na Escola, 24(1):21-30.

Coutinho, E. F., Santos, I., and Bezerra, C. I. M. (2017a). A software ecosystem for a virtual learning environment: Solar seco. In Proceedings of the Joint 5th International Workshop on Software Engineering for Systems-of-Systems and 11th Workshop on Distributed Software Development, Software Ecosystems and Systems-of-Systems.

Coutinho, E. F., Santos, I., Moreira, L. O., and Bezerra, C. I. M. (2019). A report on the teaching of software ecosystems in software engineering discipline. In Proceedings of the XXXIII Brazilian Symposium on Software Engineering, SBES 2019.

Coutinho, E. F., Viana, D., and dos Santos, R. P. (2017b). An exploratory study on the need for modeling software ecosystems: The case of solar seco. In 9th International Workshop on Modelling in Software Engineering (MISE), MISE '17, pages 47-53.

Iansiti, M. and Levien, R. (2004). Strategy as ecology. Harvard business review, 82(3):68-78, 126.

Jansen, S., Brinkkemper, S., and Finkelstein, A. (2009). Business network management as a survival strategy: A tale of two software ecosystems. In Proceedings of the First International Workshop on Software Ecosystems (IWSECO-2009), co-located with the 11th International Conference on Software Reuse, volume 505. CEUR-WS.org.

Manikas, K. (2016). Revisiting software ecosystems research: A longitudinal literature study. Journal of Systems and Software, 117:84 - 103.

Manikas, K. and Hansen, K. M. (2013). Software ecosystems - a systematic literature review. J. Syst. Softw., 86(5):1294-1306.

Mizushima, K. and Ikawa, Y. (2011). A structure of co-creation in an open source software ecosystem: A case study of the eclipse community. In 2011 Proceedings of PICMET '11: Technology Management in the Energy Smart World (PICMET).

Santos, R., Valença, G., Viana, D., Estácio, B., Fontão, A., Marczak, S., Werner, C., Alves, C., Conte, T., and Prikladnicki, R. (2014). Qualidade em ecossistemas de software: Desafios e oportunidades de pesquisa. In Proceedings of VIII Workshop on Distributed Software Development, Software Ecosystems and Systems-of-Systems, pages 41-44.

UNESCO (2021). Draft text of the unesco recommendation on open science. https://unesdoc.unesco.org/ark:/48223/pf0000376893?posInSet=7\&queryId=64f6c09b9508-4258-82a1-e195d9d38368. Online; acessado em julho-2021. 